\title{
Purge flow effects on rotor hub endwall heat transfer with extended endwall contouring into the disk cavity
}

\author{
Original article \\ Article history: \\ Accepted: 2 April 2019 \\ Published: 13 May 2019 \\ This paper is the updated version of \\ a paper originally presented at the Global \\ Power and Propulsion Technical \\ Conference, GPPS Zurich19, in Zurich, \\ Jan 15-16 2019.
}

\section{Check for updates}

\section{*Correspondence:}

DDH: dohaenni@ethz.ch

\section{Peer review:}

Single blind

\section{Copyright:}

(c) 2019 Hänni et al. @ This is an open access article distributed under the Creative Commons Attribution Non Commercial No Derivatives License (CC BY-NC-ND 4.0). Unrestricted use, distribution, and reproduction of the original work are permitted for noncommercial purposes only, provided it is properly cited and its authors credited. No derivative of this work may be distributed.

\section{Keywords:}

heat transfer; rotor endwall; purge flow; endwall contouring; infrared thermography

\section{Citation:}

Hänni D. D., Schädler R., Abhari R. S., Kalfas A. I., Schmid G., Lutum E., and Lecoq N. (2019). Purge flow effects on rotor hub endwall heat transfer with extended endwall contouring into the disk cavity. Journal of the Global Power and Propulsion Society. 3: 555-568. https://doi.org/10.33737/jgpps/109838

\author{
Dominic D. Hänni ${ }^{1, *}$, Rainer Schädler ${ }^{1}$, Reza S. Abhari ${ }^{1}$, Anestis I. Kalfas ${ }^{2}$, \\ Gregor Schmid ${ }^{3}$, Ewald Lutum ${ }^{4}$, Nicolas Lecoq ${ }^{4}$ \\ ${ }^{1}$ ETH Zurich, Sonneggstrasse 3, ML J 33, Zürich 8092, Switzerland \\ ${ }^{2}$ Aristotle University of Thessaloniki, Thessaloniki 54124, Greece \\ ${ }^{3}$ Siemens AG, Mellinghofer Str. 55, Muelheim an der Ruhr 45473, Germany \\ ${ }^{4}$ MTU Aero Engines AG, Dachauer Str. 665, München 80995, Germany
}

\begin{abstract}
Efficiency improvements for gas turbines are strongly coupled with increasing turbine inlet temperatures. This imposes new challenges for designers for efficient and adequate cooling of turbine components. Modern gas turbines inject bleed air from the compressor into the stator/ rotor rim seal cavity to prevent hot gas ingestion from the main flow, while cooling the rotor disk. The purge flow interacts with the main flow field and static pressure field imposed by the turbine blades. This complex interaction causes non-uniform and jet-like penetration of the purge flow into the main flow field, hence affecting the endwall heat transfer on the rotor.

To improve the understanding of purge flow effects on rotor hub endwall heat transfer, an unshrouded, high-pressure representative turbine design with 3D blading and extended endwall contouring of the rotor into the cavity seal was tested. The measurements were conducted in the 1.5-stage axial turbine facility LISA at ETH Zurich, where a state-of-the-art measurement setup with a high-speed infrared camera and thermally managed rotor insert was used to perform high-resolution heat transfer measurements on the rotor. Three different purge flow rates were investigated with regard to hub endwall heat transfer. Additionally, steady-state computational fluid dynamics simulations were performed to complement the experiments.

It was found that the local heat transfer rate changes up to $\pm 20 \%$ depending on the purge flow rate. The main part of the purged air is ejected at the endwall trough location and swept towards the rotor suction side, which is caused by the interaction of main flow and the cavity extended endwall design. The presence of low momentum purge flow locally reduces the heat transfer rate. Changes in adiabatic wall temperature and heat transfer (depending on purge rate) are observed from the platform start up to the cross passage migration of the secondary flow structures.
\end{abstract}

\section{Introduction}

Over the past years, various design approaches have been developed to increase the efficiency and reduce losses due to secondary flows in turbomachines. According to (Sharma and Butler, 1987), up to 30\%-50\% of the total aerodynamic losses can be attributed to secondary flow losses. (Langston, 2001) provides a condensed review about secondary flows in turbomachines. 
Endwall contouring is a promising approach for reducing and controlling the cross passage flow due to the pressure gradient. Studies by (Harvey et al., 1999) showed that the usage of non-axisymmetric endwall contouring is a powerful tool for reducing secondary flows with comparable effects to the lean and skew of blades. Cascade measurements confirmed a reduction of secondary losses by 30\% (Hartland et al., 1999). Efficiency increases of $1.0 \%$ and $0.4 \%$ were also reported by (Schuepbach et al., 2008) and (Snedden et al., 2009), respectively.

For high-pressure turbines, the presence of cavity leakage flows for cooling the rotor disk and preventing hot gas ingestion is both omnipresent and required. Measurements by (Jenny et al., 2013) showed that the presence of purge flow increases the unsteadiness, and increases the rotor hub passage losses by $4 \%$ for each percent of purge flow injected. The beneficial effect of endwall contouring was also confirmed by (Regina et al., 2014). However, they found that endwall contouring increases the sensitivity of stage efficiency to purge flow injection, and eventually the beneficial impact is lost. Similar results were reported by (Schuepbach et al., 2010), where a reduction of $1.2 \%$ in efficiency per percent of purge injection was found.

The effect of purge flow and endwall contouring on heat transfer is mainly investigated in linear cascades. (Thrift et al., 2012) showed that a slot angle of $45^{\circ}$ increases cooling effectiveness (compared to $90^{\circ}$ ) due to a different formation of the horseshoe vortex. An overall averaged reduction in Nusselt number for the endwall, both with and without purge flow, was reported by (Roy et al., 2017) for a contoured endwall due to secondary flow control. Further, (Lynch et al., 2010) reported a 3.1\% reduction of averaged heat transfer for endwall contouring. However, a local increase was observed around the blade leading edge region (where the horseshoe vortex forms), and a decrease was observed at the suction side of the blade. The same authors reported that increased purge flow rates increased the heat transfer on the suction side due to strengthening of the vortical flow structures. An area-averaged increase of $8 \%$ was reported for heat transfer, and a $27 \%$ increase in cooling effectiveness.

Heat transfer measurements in rotating facilities are not widely available in open literature. Time resolved data was presented (among others) by (Dunn et al., 1984) and (Abhari et al., 1992) using thin film heat flux gauges. Endwall heat transfer measurements in a rotating facility were first presented by (Blair, 1992). Recently, (Lazzi Gazzini et al., 2017b) presented high-resolution heat transfer measurements using high-speed infrared thermography on the rotor endwall, taken in the same facility as used for this study. They found that the purge flow influences the Nusselt number distribution on the suction side of the rotor passage, up to approximately $30 \%$ axial extent. However, the measured differences were within the measurement uncertainty.

Previous studies have focused on endwall contouring within the rotor passage or purge flow injection separately. This work investigates an endwall design that is extending into the cavity; hence, strongly interacting with the purge flow. High resolution experimental data is presented from measurements in a rotating facility.

\section{Methodology}

\section{Research facility and operating conditions}

The data presented in this paper is based on experiments conducted in the axial turbine research facility LISA of the Laboratory for Energy Conversion (LEC) at ETH Zurich, as depicted in Figure 1. The test rig is a lowtemperature, moderate-speed, continuously running rotating facility with non-dimensional parameter matching of real engine conditions. A radial compressor drives the air in the quasi-closed loop trough a two-stage water to air heat exchanger and a flow conditioning stretch before the test section. The turbine inlet total temperature is controlled within an accuracy of $\pm 0.4 \mathrm{~K}$. After the turbine section, the loop is open to the atmosphere, and the flow is then fed back through a calibrated Venturi nozzle for mass flow measurements to the compressor. The turbine shaft is connected to a reduction gearbox and a DC generator, breaking the turbine and controlling the rotational speed within an accuracy of $\pm 0.02 \%( \pm 0.5 \mathrm{rpm})$. The generated power is then fed back to the power grid. Because the air loop is open to the atmosphere, two rows of subsequent de-swirling vanes are installed to recover the static pressure back to ambient levels. A more detailed description of the rig and test section is given by (Behr et al., 2006).

The investigated turbine configuration is a 1.5-stage, high-pressure turbine representative design. All blade rows and endwalls are $3 \mathrm{D}$ optimised geometries for reduced aerodynamic losses. The blade count for stator 1 , the rotor, and stator 2 are 36, 42, and 36, respectively.

Compressor air from the main loop is bypassed and used as cavity purge flow in the rotor upstream cavity. The air is extracted before the flow stretch, and injected through 10 tubes in the first stator vanes into the cavity 


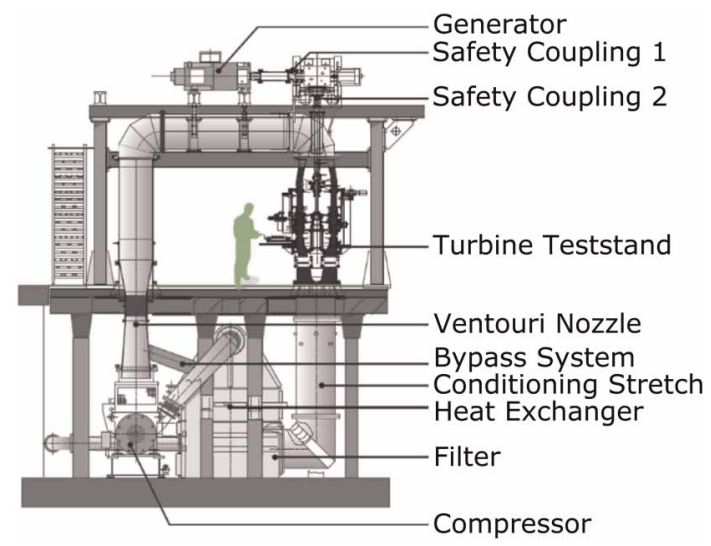

Figure 1. Overview of the turbine test facility "LISA" at ETH Zurich.

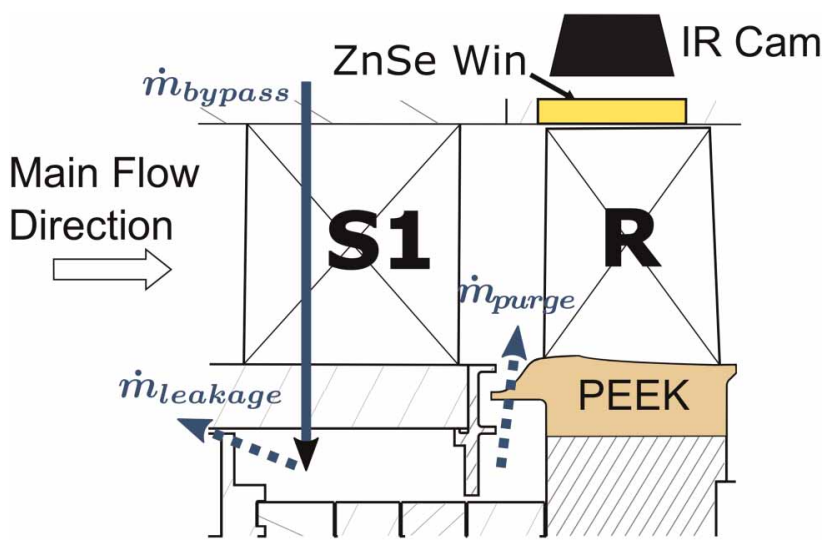

Figure 2. Schematic cut of first stage with purge flow injection path and optical access to the rotor endwall.

below. The purge flow injection rate (IR) from the cavity into the main annulus is defined as follows:

$$
I R=\frac{\dot{m}_{\text {bypass }}-\dot{m}_{\text {leakage }}}{\dot{m}_{\text {tot }, \text { turbine }}} \cdot 100
$$

The purge air is calculated from the mass flow balance of extracted bypass flow and cavity sealing leakage flow through the drum, normalised by the total turbine mass flow (Figure 2). Both mass flow rates are measured with calibrated standard nozzles.

For this study, the turbine was operated at the constant design pressure ratio of $\Pi_{1.5}=1.65$, accounting for changes in atmospheric pressure. Further relevant operating parameters are summarised in Table 1.

\section{Rotor endwall and cavity sealing geometry}

The rotor upstream cavity sealing is an overlapping angle-wing type with $2.1 \% c_{a x}$ of overlapping (Figure $3 \mathrm{~b}$ ). Additionally, a radial sealing arm separates the injection plenum from the rotor disk. This sealing geometry is representative of real engine designs.

The non-axisymmetric rotor hub endwall contouring is extended upstream into the rotor sealing arm arrangement. The contouring starts at $-23 \% c_{a x}$ upstream of the rotor leading edge, at a radial location inside the sealing arrangement below the nominal hub radius of $330 \mathrm{~mm}$. The contouring consists of a pronounced hill on the pressure side of the passage next to a trough at the suction side, near the blade leading edge axial location

Table 1. Rig operation conditions.

\begin{tabular}{|l|l|l|}
\hline Parameter & Value & Units \\
\hline Total rotor relative inlet temperature $T_{t, r e l}$ & $313.5 \pm 0.1$ & {$[\mathrm{~K}]$} \\
\hline Pressure ratio $\Pi_{1.5}$ & $1.65 \pm 0.4 \%$ & {$[-]$} \\
\hline Rotor Inlet Mach number & 0.26 & {$[-]$} \\
\hline Mass flow & 11.7 & {$[\mathrm{~kg} / \mathrm{s}]$} \\
\hline Rotor speed & 2700 & {$[\mathrm{rpm}]$} \\
\hline Purge Injection Ratio $I R$ & $0.0 \% / 0.8 \% / 1.2 \%$ & {$[-]$} \\
\hline Purge cavity temperature $T_{C}$ & $326.4 / 325.4 / 324.2$ & {$[\mathrm{~K}]$} \\
\hline
\end{tabular}



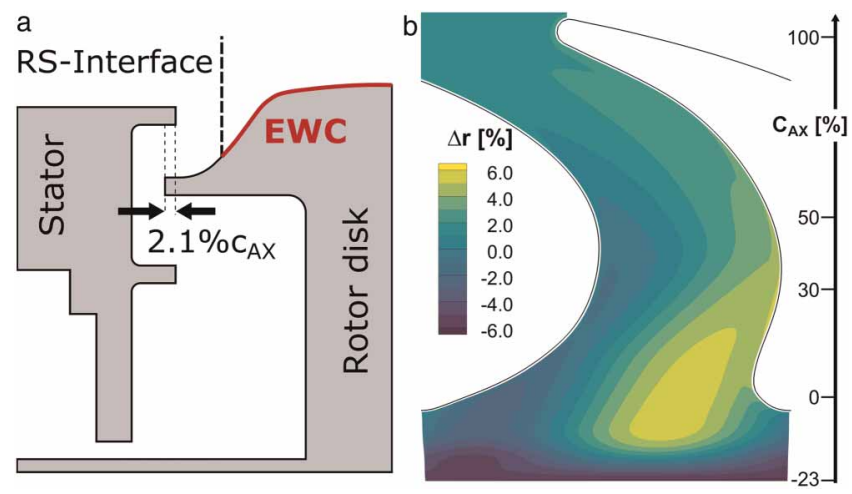

Figure 3. Rotor stator hub cavity sealing configuration (a) and hub endwall contouring (b).

(Figure 3b). As shown, most of the contouring is located upstream of the leading edge until reaching $50 \%$ of the axial chord. The rotor blades have a constant fillet radius of $3 \mathrm{~mm}$.

\section{Design intention of rotor endwall}

The design of the rotor endwall contouring is based on a CFD optimisation cycle, which only uses the 1.5-stage aerodynamic efficiency as an optimisation target, with mass flow as a constrain. The design steps were as follows:

(1) The geometry was created using CAESES by Friendship Systems. The design of the non-axisymmetric endwall required only four parameters. Hill and trough were both specified in terms of amplitude, axial position, axial extent, and circumferential propagation relative to the camber line of the aerofoil. The endwall was optimised together with the aerofoil by adding these additional parameters to the converged optimisation setup of the blade.

(2) A block-structured mesh was generated with AutoGrid by Numeca. The domain included three blade rows and the rotor upstream cavity. To save computational cost, only the rotor blade row was re-meshed during the design optimisation. A butterfly topology was used to generate a fillet radius between the aerofoils and the non-axisymmetric endwall. The main gas path was modified such that it dipped into the cavity for two reasons: (a) The radius at the platform leading edge can be modelled correctly and (b) the non-conformal interface between cavity and rotor blade (RS-Interface) is shifted upstream, allowing for an extension of the endwall contouring (EWC) into the cavity (Figure 3a).

(3) The steady CFD simulations were conducted using TRACE, a code specifically developed for turbomachinery applications by the Institute of Propulsion Technology at DLR. Radial distributions of total quantities and static pressure from previous measurements served as inlet and outlet boundary conditions. The purge air was specified by a mass flow boundary condition of $0.8 \%$ of the main flow, and turbulence was modelled with the shear stress transport model (SST).

This toolchain was run with AutoOpti by DLR, using a generic algorithm for the generation of designs and a surrogate model based on kriging. In total, 89 design parameters were used for the aerofoil and endwall. The optimisation required approximately 1,500 members to converge. More details about the optimisation algorithm are given in (Aulich and Siller, 2011).

A difference in efficiency of 0.3 percentage points in 1.5-stage efficiency compared to flat endwall was achieved in the optimisation. However, as the aerofoil and endwall were optimised in combination, it was difficult to isolate the effect of the EWC itself. Compared to a flat endwall, a more uniform mass distribution of the purge over the circumference was observed. Further, the cross-passage migration of the pressure side horseshoe leg shifted downstream, and the passage vortex and suction side horseshoe vortex leg remain separated. This resulted in two small pressure loss cores, compared to one big loss core without contouring.

\section{Experimental setup}

Heat transfer measurements in the facility were performed with an infrared based technique described by (Lazzi Gazzini et al., 2017b) and (Laveau et al., 2014). A Flir IR SC7300L camera was used to record the temperature of the rotor endwall through infrared transparent Zinc-Selenide $(\mathrm{ZnSe})$ windows installed in the rotor casing (Figure 2). The camera was mounted on a traversing system, allowing vertical adjustments and different camera angles using various angle shims to extend the optical coverage through the window. 
A quasi iso-energetic setup was used to create controlled boundary conditions at the region of interest. Therefore, a custom-made thin film surface heating insert was manufactured and installed on the rotor. The insert was manufactured from PEEK (Polyether-ether-ketone), a thermally low conductive, high strength, base substrate, replicating the exact endwall geometry. Pt100 temperature sensors were installed in the insert $1.5 \mathrm{~mm}$ below the surface to account for conduction losses. The surface was coated with an $1.4 \mu \mathrm{m}$ thick layer of Nickel using a direct chemical deposition technique on the PEEK, and pulsed laser ablation was used to create a serpentine like heating track with a uniform width of $1.9 \mathrm{~mm}$. The heating track on the endwall is depicted in Figure 4a, also indicating the achieved heated area in red. The inherent need for track turns resulted in unheated areas close to the blade fillet, which were therefore excluded in the post-processing. For improved infrared signature and reduction of reflections, all rotor surfaces were painted with Nextel black velvet high emissivity paint $(\varepsilon=0.98$ (Kwor and Matteï, 2001)).

Nonuniformities in heat flux creation were corrected during post-processing with the non-uniformity factor $S_{q}$ derived from transient heating tests, as described by (Lazzi Gazzini et al., 2017a) and defined in Equation 2. This local factor accounts for differences in electrical resistance or current concentrations on the heater.

$$
S_{q}=\frac{\dot{q}_{e l, l o c}^{\prime \prime}}{\dot{q}_{e l, a v g}^{\prime \prime}}
$$

The temperature sensor data acquisition and control of the heaters was accomplished with a rotating data acquisition system mounted on the rotor disk, controlled over a wireless connection (Mansour et al., 2015). The electrical power for the heating platforms (and for the rotor on-board data acquisition system) was transferred to the rotating system via a two-channel slip-ring. The heater supply current was measured and recorded externally.

\section{Image processing}

Measurements were performed for a specific stator 1 to rotor relative position. A shaft mounted optical triggering system was used to trigger the infrared camera acquisitions, and images were recorded with a sensor integration time of $10 \mu$ s to reduce the blurring due to platform movement. For extended optical coverage of the endwall, three different camera tilting angles were used $\left(-4.5^{\circ},+4.5^{\circ}\right.$, and $\left.+15^{\circ}\right)$, and a sequence of 1,000 individually triggered images were recorded for each position. All frames of an image sequence were registered with respect to a reference, and only images within a \pm 1 pixel shift were considered for post-processing. Three different platform power levels, with an averaged produced heat flux between 3.3 and $9.2 \mathrm{~kW} / \mathrm{m}^{2}$, were used for the calculation of heat transfer quantities.

Silver infrared reference markers were used on the endwall to project the two dimensional image data on the three dimensional endwall geometry by geometrical transformation.

\section{Heat transfer calculation}

The convective heat flux is calculated from the overall energy balance (Equation 3) on the heater surface, schematically depicted in Figure 4b.

$$
\dot{q}_{c o n v}^{\prime \prime}=\dot{q}_{e l}^{\prime \prime}-\dot{q}_{c o n d}^{\prime \prime}-\dot{q}_{r a d}^{\prime \prime}
$$

The electric heat flux is produced by Joule heating (Equation 4) and directly calculated from the supply voltage and current and corrected for local non-uniformity by the factor $S_{q}$. The radiative heat flux is calculated

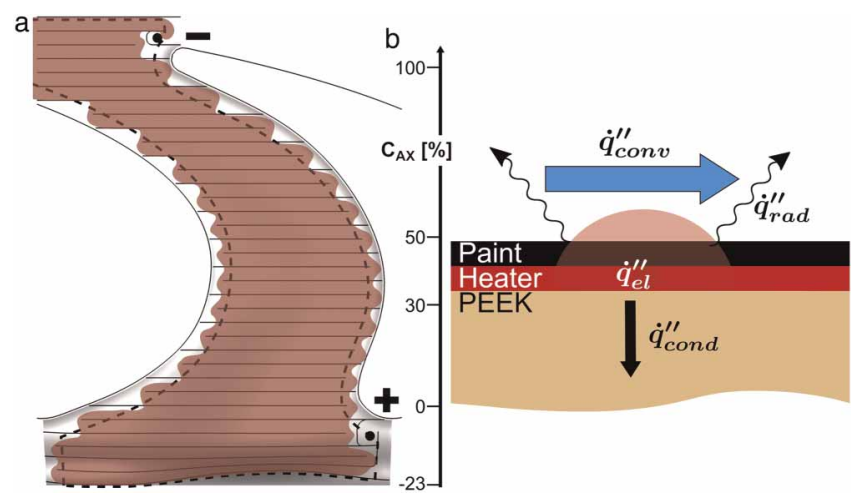

Figure 4. Endwall heating track design with indicated heated area (a) and energy balance of the setup (b). 
according to Equation 5 with the emissivity $\varepsilon$ of the paint and $\sigma$ being the Stefan-Boltzmann constant. The conduction losses are calculated using the installed temperature sensors inside the heater, according to the one dimensional conduction Equation (6) and based on the thermal properties of the substrate.

$$
\begin{aligned}
\dot{q}_{e l}^{\prime \prime} & =S_{q} \frac{V \cdot I}{A_{\text {surf }}} \\
\dot{q}_{\text {rad }}^{\prime \prime} & =\varepsilon \cdot \sigma \cdot\left(T_{w}^{4}-T_{t, r e l}^{4}\right) \\
\dot{q}_{\text {cond }}^{\prime \prime} & =\frac{k_{\text {sub }}}{d_{\text {sub }}} \cdot\left(T_{w}-T_{\text {sub }}\right)
\end{aligned}
$$

The conduction losses were calculated for every heat flux step, and averaged over the passage for the energy balance.

For each heating step, the convective heat flux and wall temperature were obtained. A least square linear fitting was used to calculate the local heat transfer coefficient $\mathrm{h}$ and adiabatic wall temperature $T_{a w}$ (as defined in Equation 7) from the three heating steps.

$$
\dot{q}_{c o n v}^{\prime \prime}=h \cdot\left(T_{w}-T_{a w}\right)
$$

\section{Numerical setup}

A multi-block structured mesh was generated for the 1.5 stage configuration (including cavities). The mesh consisted of approximately 11.6 million nodes, which provided a high-quality boundary layer resolution. The distribution of the non-dimensional wall distance $y^{+}$for the simulation domain is shown in Figure 5.

The numerical investigation was performed with the commercial solver ANSYS CFX version 17.0. Steady state computations were conducted using the high-resolution advection scheme and the total energy equation, including viscous work terms for compressible flow. Computations were performed with the SST turbulence model with the $\gamma-\theta$ transition model and mixing plane for the stator-rotor interface.

The surface roughness from the experiments was modelled using the roughness model supplied in CFX. Details about the model are described by (Lutum et al., 2015). Local roughness quantities were derived by profilometer measurements along the blade endwall. From this investigation, an equivalent sand grain roughness level of $20 \mu \mathrm{m}$ was determined and applied for the current predictions. The spatial discretisation was quasi-second-order for all investigated cases.

Inlet profiles for total pressure, total temperature, and turbulence quantities were obtained from corresponding experiments. The purge flow boundary conditions were specified by a mass flow rate of IR $=0.8 \%$ and total temperature of $328 \mathrm{~K}$. Numerical heat transfer results were determined from a set of computations consisting of an adiabatic and heat flux calculation for the same operating conditions.

\section{Results and discussion}

Distributions of heat transfer quantities are shown for the three different purge flow injection ratios, as defined in Table 1. The presented results were smoothed (to reduce noise and clarify the data) by using 30 passes of Tecplot 360 smoothing with a relaxation factor of 0.5 .

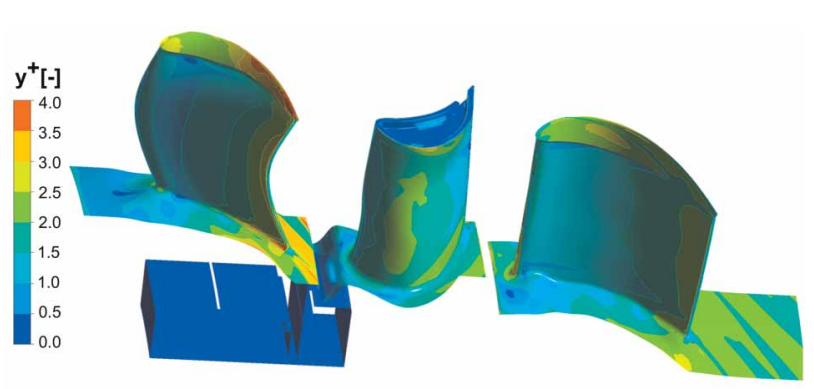

Figure 5. Non-dimensional wall distance for CFD simulations. 
The local heat transfer coefficient is represented as Nusselt number, as defined in Equation 8:

$$
N u=\frac{h \cdot c_{a x}}{k_{a i r}}
$$

where $c_{a x}$ is the rotor axial chord at mid-span, and $k_{a i r}$ is the conductivity of air. The adiabatic wall temperature is presented for the design case in a non-dimensional form given in Equation (9).

$$
\theta=\frac{T_{a w}-T_{t, r e l}}{\frac{w^{2}}{2 \cdot c_{p}}}
$$

All flow reference quantities are mid-span circumferentially mass-averaged values at the rotor inlet. Values were taken from experimental probe measurements with the LEC in-house manufactured fast response aerodynamic probe (FRAP), performed during the same measurement campaign. More details on the FRAP probe and measurement techniques can be found in (Kupferschmied et al., 2000) and (Pfau et al., 2003).

\section{Measurement uncertainty}

The measurement uncertainty evaluation was performed by successively perturbing the input parameters in the post-processing routine as sequential perturbation, as described by (Moffat, 1988). The local distribution of the Nusselt number and non-dimensional adiabatic wall temperature uncertainty for the design case are presented in Figure 6.

For the measurements presented, the platform averaged relative uncertainty was reported to be $\pm 12.0 \%$ for the Nusselt number, and an absolute uncertainty of \pm 0.14 [-] for the non-dimensional adiabatic wall temperature. The main contributors to the Nusselt number uncertainty were the conductive losses (67.9\%), the surface temperature $(27.2 \%)$, and non-uniformity scaling factor $S_{q}(3.5 \%)$. For the adiabatic wall temperature, the uncertainty in surface temperature $(70.3 \%)$ and conduction losses $(29.7 \%)$ were the main contributors. These uncertainty contributions are summarised in Table 2.

\section{Endwall heat transfer for design point operation}

Figure 7 shows the Nusselt number and adiabatic wall temperature distribution on the hub endwall for the design purge condition IR $=0.8 \%$.

The small band of very high heat transfer at the beginning of the heated platform was consistent with results reported previously with a similar measurement setup (Lazzi Gazzini et al., 2017b; Laveau et al., 2013), and arose from the start of the thermal boundary layer. A wedge like region of increased heat transfer followed at the mid-passage position, extending to approximately $10 \% c_{a x}$ from the leading edge measured at the mid-span (region A). This region coincides with the hill of the endwall contouring (Figure $3 \mathrm{~b}$ ) and can be explained by the induced acceleration of the flow. In contrast, lower heat transfer was observed towards the leading edge suction side of the blade, following the endwall trough. The isolated small region upstream of the leading edge, with very low heat transfer, was introduced by the electrical connection of the thin film heater, which was

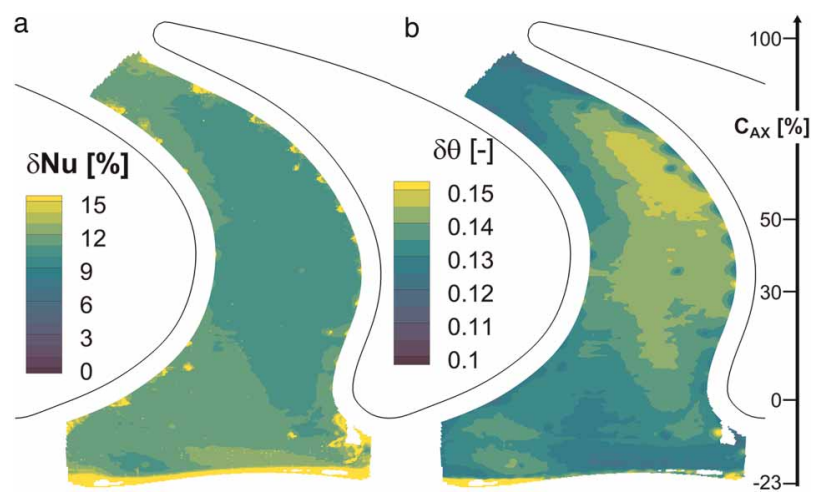

Figure 6. Uncertainty distributions of relative Nusselt number (a) and absolute adiabatic wall temperature (b). 
Table 2. Relative measurement uncertainty contributions.

\begin{tabular}{|l|c|c|c|c|}
\hline & $\delta T_{w}$ & $\delta \dot{q}_{\text {cond }}^{\prime \prime}$ & $\delta S_{q}$ & $\delta l$ \\
\hline$\delta N u$ & $27.2 \%$ & $67.8 \%$ & $3.5 \%$ & $1.5 \%$ \\
\hline$\delta \theta$ & $70.2 \%$ & $29.7 \%$ & $0.0 \%$ & $0.0 \%$ \\
\hline
\end{tabular}

neglected in the analysis. Moreover, an increase in heat transfer was observed towards the throat region, which was attributed to the flow acceleration.

The adiabatic wall temperature (Figure $7 \mathrm{~b}$ ) decreased through the rotor passage. This was expected, as the flow temperature decreases due to expansion and acceleration. As the bypass air was extracted upstream of the turbine, the injected purge flow had a higher temperature than the main flow. Therefore, contrary to cooling in real gas turbines, the purge flow was heating the endwall in this measurement setup. The very start of the platform exhibited the highest adiabatic wall temperature over the full pitch, with iso-lines closely following the shape of the endwall contouring (Figure 3b).

As this part of the endwall extends inside the cavity and below the hub radius of the upstream NGV, it can be concluded that the changes seen in this region are associated with purge flow interaction. The extent of increased adiabatic wall temperature at the suction side (up to the $30 \%$ axial chord) indicates that a high amount of hot purge air is entrained into the suction side horseshoe vortex, whereas the rapid decrease over the endwall hill indicates the presence of the main flow.

\section{Comparison with CFD predictions}

The predictions from CFD simulations were compared to the experimental data by means of the endwall resolved Nusselt number and non-dimensional adiabatic wall temperature distributions, and the lateral averaged data of these values.

The simulation results for design purge flow rate are depicted in Figure 8. The increase of local heat transfer at the endwall hill upstream of the leading edge was clearly captured by CFD, which was visible as a wedge-like region (region A). The effect of the horseshoe vortex at the pressure side leading edge (region B) was not as clearly visible in the experiments as in CFD. However, a local increase of heat transfer was observed in both cases. No influence of the horseshoe vortex on the suction side was visible for both the experiments and the simulation, as the effect was too close to the blade and therefore not covered by the heated surface of the measurement setup. The adiabatic wall temperature exhibited two locations of purge entrainment into the passage with increased temperature (regions $\mathrm{A}$ and $\mathrm{B}$ ). The larger region following the side of the endwall, which extended up to $30 \% c_{a x}$, was similar to the hot streak in the experiments (Figure $7 \mathrm{~b}$ ) except for a slight pitchwise shift. The second region (B) was not visible in the experiments. The relevant patterns for both quantities were captured in the simulation.

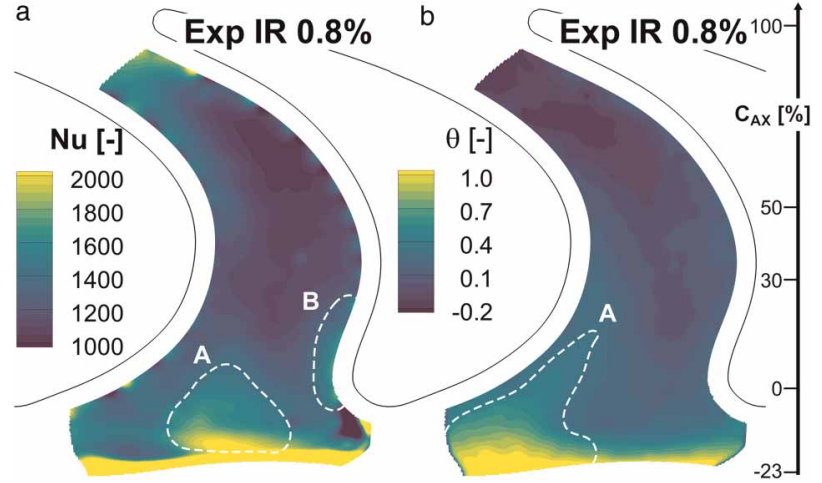

Figure 7. Nusselt number (a) and adiabatic wall temperature (b) distributions for design purge flow rate (IR $0.8 \%$ ).

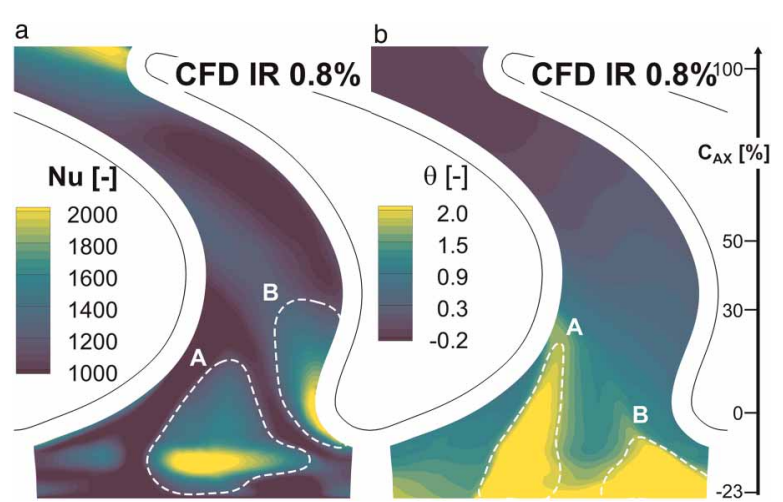

Figure 8. Distributions from CFD simulations of Nusselt number (a) and adiabatic wall temperature (b) for IR $0.8 \%$. 
The lateral averaged results are compared in Figure 9. A general agreement within $\pm 15 \%$ for the Nusselt number was found, although the high heat transfer region at the start of the heater was not captured by the simulations. Further, the increase through the passage was predicted to be less pronounced and start farther downstream than measured in the experiment. The trends for the adiabatic wall temperature were captured with a change in slope at approximately $-5 \% c_{a x}$. The offset in adiabatic wall temperature in the simulation, especially at the beginning of the platform, is explained by a miss-match in purge flow temperature and a different heat pickup compared to the experiments.

\section{Purge flow influence on endwall heat transfer}

The effect of purge flow on the endwall heat transfer was investigated with three different injection rates. Accordingly, the design point IR $=0.8 \%$ case was the reference, and compared against zero injection $\mathrm{IR}=0.0 \%$ and a high injection rate $I R=1.2 \%$. This was accomplished by means of the relative Nusselt number difference, defined as follows:

$$
\Delta N u=\frac{N u_{I R}-N u_{I R 0.8 \%}}{N u_{I R 0.8 \%}}
$$

Figure 10 shows the relative difference to nominal injection for both off-design cases. A general observation reveals that for both cases, there were local changes in heat transfer up to $\pm 20 \%$. However, significant changes were only found within the first $30 \%$ of the axial chord length. This implies that after the passage cross flow hits the blade suction side, all the purge flow is mixed into the secondary flows and detached from the endwall.

In the case of zero purge injection, a distinct increase of heat transfer was observed along the suction side, from the leading edge downstream to approximately $c_{a x}=0.3$. This implies that the purge flow in the reference case interacts with the suction side horseshoe vortex, which usually enhances the local heat transfer due to increased shear. However, for the investigated geometry with endwall contouring extending into the cavity, the purge flow exited the cavity close to the blade suction side, as shown in Figure 11a by CFD. The low momentum purge flow was reducing the local flow velocity, and therefore the heat transfer. Over the endwall hill region, a slight decrease in heat transfer was observed for no purge flow. Additionally, the trace of increased heat transfer (starting from the pressure side leading edge over the endwall to approximately $50 \% c_{a x}$ ) indicates a stronger influence of the horseshoe vortex migration for the case without purge flow injection.

For the case of high purge flow rate, increased heat transfer was observed in a triangular region extended up to $35 \%$ of the axial chord (where it ends at the blade suction side). The highest increase (up to 20\%) was located in a band like region starting from the beginning of the platform along the side of the endwall hill.

For $\mathrm{IR}=1.2 \%$, the purge flow was entrained as two different jets into the main flow and endwall (Figure 11b). Due to the higher purge momentum, the relative yaw angle of the main flow was reduced by the purge, and enhanced mixing of both flows was present. Additionally, the purge entrainment at the suction side caused a mass redistribution and flow acceleration of the main flow in the region between the two purge jets.

The influence of purge rate on the pitch wise distribution of absolute heat transfer over the passage is shown in Figure 12, for the indicated dashed line in Figure 10. Towards the suction side, both purge flow rates of
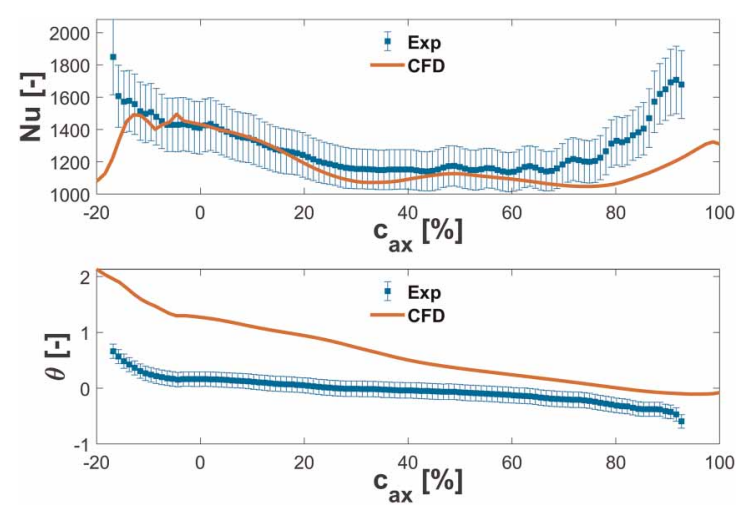

Figure 9. Lateral averaged Nusselt number (top) and adiabatic wall temperature (bottom) for CFD and experiments (IR 0.8\%).

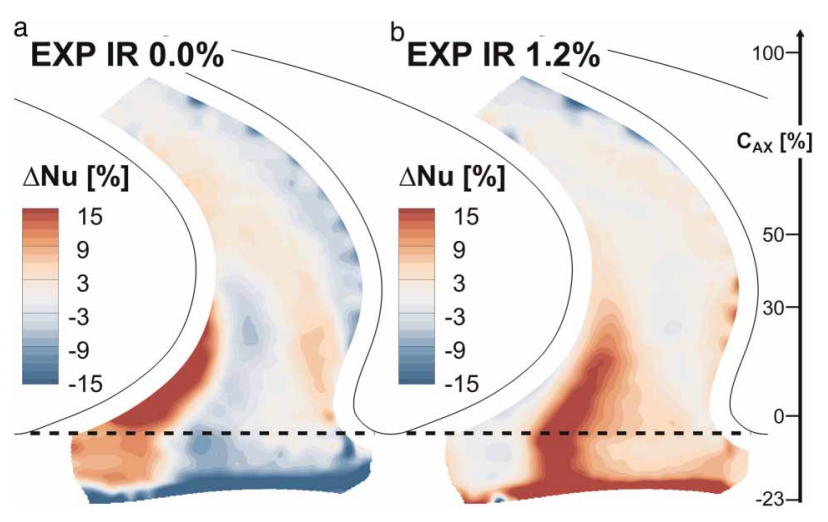

Figure 10. Relative difference in local Nusselt number for IR $0.0 \%$ (a) and IR $1.2 \%$ (b) compared to design point (IR $0.8 \%$ ). 


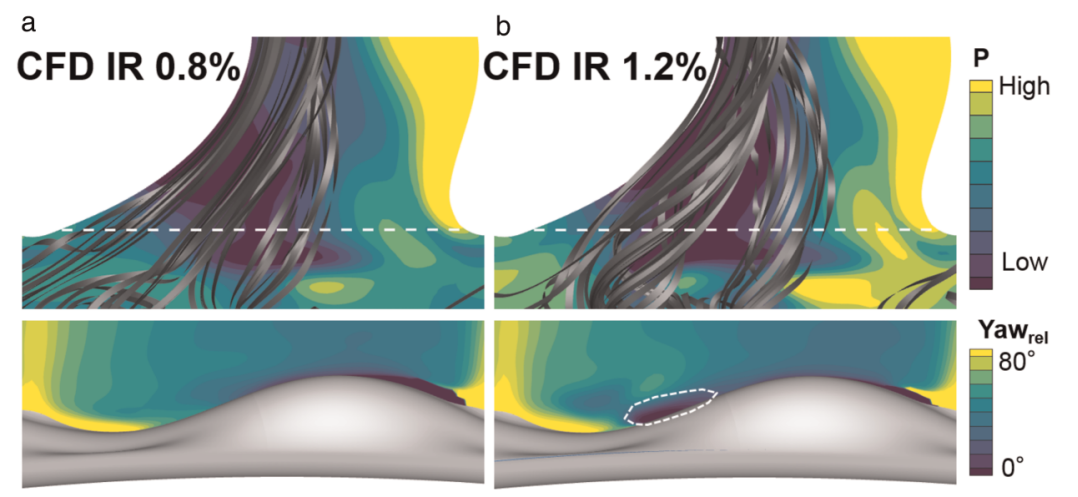

Figure 11. Purge flow stream lines (top) and relative yaw angle contour (bottom) for IR $0.8 \%$ (a) and IR $1.2 \%$ (b).

$0.8 \%$ and $1.2 \%$ exhibited near identical levels of heat transfer, whereas the zero purge rate exhibited the same trend but with elevated levels. This reduction in heat transfer was caused by the local reduction in velocity due to the presence of low momentum purge air, as described previously. Between the 0.3 and 0.6 rotor pitches, the heat transfer was the highest modulated due to purge flow. Moreover, the most uniform circumferential heat transfer distribution on the endwall was achieved without purge flow. The modulation due to purge flow was similar for both injection ratios $(0.8 \%$ and $1.2 \%)$, with different amplitudes at the maximum level leading to an increased non-uniformity over the pitch.

\section{Purge flow endwall cooling effectiveness}

The cavity purge flow injection affected the main and secondary flow structures in the rotor passage, and provided cooling of the endwall due to the temperature difference compared to the main flow. This cooling was evaluated by means of a cooling effectiveness $\eta$, as defined in Equation (11), where the adiabatic wall temperature of the zero purge flow case was taken as a reference.

$$
\eta=\frac{T_{a w}-T_{a w, I R 0.0 \%}}{T_{c}-T_{t, r e l}}
$$

The difference in adiabatic wall temperature was normalised by the difference between the purge flow temperature $T_{c}$ (measured in the cavity upstream of the radial sealing arm) and the rotor inlet relative total temperature.

Figure 13 shows the cooling effectiveness for both nominal and high purge rates for the rotor endwall. The general pattern is the same for both cases. The highest cooling effect was observed at the very beginning of the platform, where the endwall is below the nominal main flow hub radius (and therefore immersed in the purge flow). The other area of purge cooling started at the EWC trough and extended up to $30 \%$ of the axial chord along the blade suction side. This pattern was very similar to the regions of decreased heat transfer due to purge flow injection discussed in the previous section (Figure 9). This further confirms that the purge flow is predominantly swept to the suction side of the blade. This concentration is also illustrated in Figure 14, which shows the circumferential distribution for the same axial position indicated in Figure 13. The effectiveness is approximately twice as high at the suction side with the endwall trough compared to the rest of the pitch.

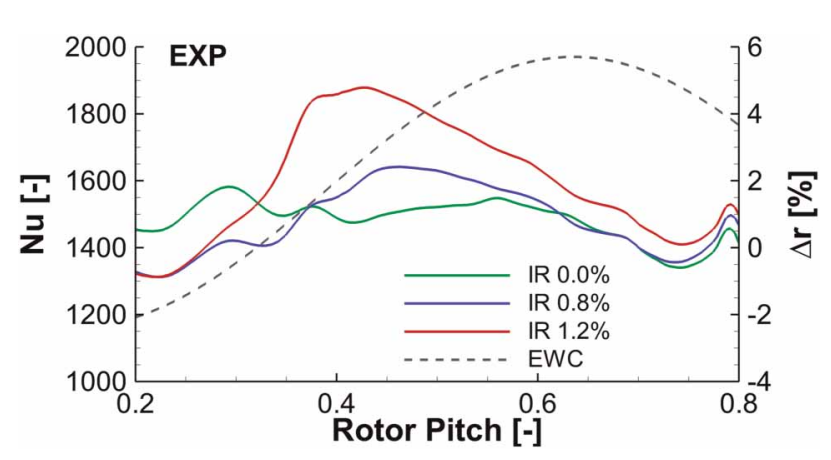

Figure 12. Pitch-wise Nusselt number distribution and endwall radius change at indicated location in Figure 10. 


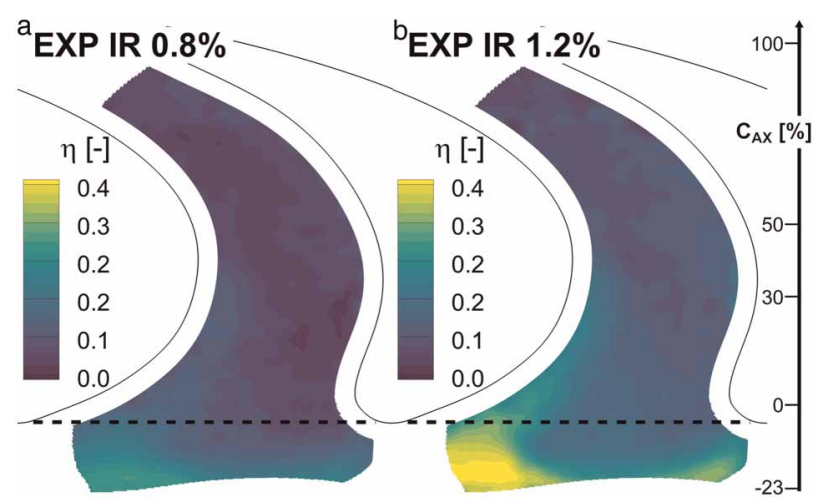

Figure 13. Experimental endwall cooling effectiveness for IR $0.8 \%$ (a) and IR $1.2 \%$ (b).

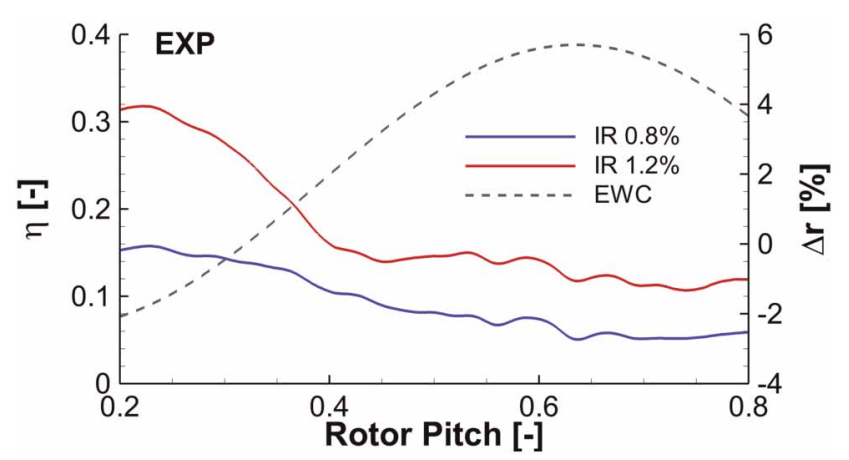

Figure 14. Pitch-wise cooling effectiveness distribution at indicated location in Figure 13.

The cooling effectiveness was approximately $75 \%$ higher for the high injection case (over the design injection case), where the overall platform averaged effectiveness was 0.085 for $\mathrm{IR}=0.8 \%$, and 0.150 for $\mathrm{IR}=1.2 \%$.

\section{Conclusions}

This work presents high-resolution heat transfer measurement data of a high-pressure representative turbine rotor endwall (with advanced non-axisymmetric endwall contouring) in a rotating turbine facility. A new technique was used to manufacture a thin film quasi-iso-energetic heating surface on arbitrarily shaped surfaces, and to create controlled thermal boundary conditions for fast infrared surface temperature measurements. The influence of different purge flow injection rates on endwall heat transfer and cooling effectiveness was investigated for a contoured endwall extended into the disk cavity.

Predictions from CFD simulations were generally in good agreement with the experiments (within $\pm 15 \%$ ), and captured the relevant local heat transfer patterns.

Cavity extended non-axisymmetric endwall contouring was shown to significantly influence the local heat transfer rate from the beginning of the platform up to $30 \%$ of the axial blade chord. Compared to the design conditions, differences of up to $20 \%$ in local Nusselt number were observed for both the no purge and high purge flow rates. Endwall resolved CFD results were required to resolve these local changes, which were not captured by lateral averaged data.

Compared to the case without purge flow, the average cooling effectiveness was 0.085 and 0.150 for purge rates of $0.8 \%$ and $1.2 \%$, respectively. Therefore, these results suggest that during the design and optimisation process of advanced geometries for highest aerodynamic efficiencies, auxiliary air injection should be included in addition to the local heat load.

Advanced tools for aerodynamic performance optimisation can lead to very specific designs. For the investigated geometry, the injection of purge flow beneficially reduces the heat transfer at the stress critical region of the blade root leading edge suction side. However, this benefit is counteracted by increased pitch-wise differences and non-uniformities over the platform. Owing to this sensitivity of heat transfer to the purge rate, off-design performance also needs to be considered during the design phase. 


\section{Nomenclature}

\section{Variables}

$c$ blade chord at mid-span [-]

$b$ heat transfer coefficient $\left[\mathrm{W} / \mathrm{m}^{2} / \mathrm{K}\right]$

I heating current $[\mathrm{A}]$

$k$ thermal conductivity $[\mathrm{W} / \mathrm{m} / \mathrm{K}]$

$N u$ Nusselt number [-]

$S_{q}$ non-uniformity heat flux scaling factor [-]

$T$ temperature $[\mathrm{K}]$

$w$ relative velocity $[\mathrm{m} / \mathrm{s}]$

$y^{+}$dimensionless wall distance [-]

\section{Greek}

$\varepsilon$ surface emissivity [-]

$\theta$ non-dimensional adiabatic wall temperature [-]

$\eta$ cooling effectiveness [-]

$\Pi$ pressure ratio [-]

\section{Subscripts}

$\begin{array}{ll}\text { aw } & \text { adiabatic wall } \\ \text { ax } & \text { axial direction } \\ \mathrm{c} & \text { coolant/purge } \\ \text { cond } & \text { conductive heat flux } \\ \text { conv } & \text { convective heat flux } \\ \text { el } & \text { Joule electric heat flux } \\ \text { rel } & \text { relative frame of reference } \\ \text { rad } & \text { radiative heat flux } \\ \text { sub } & \text { substrate } \\ t & \text { stagnation flow quantity } \\ w & \text { wall }\end{array}$

\section{Abbreviations}

CFD Computational Fluid Dynamics/Simulation

DLR Deutsches Zentrum für Luft- und Raumfahrt

EWC endwall contouring

EXP Experimental

FRAP fast response aerodynamic probe

IR injection rate

LEC Laboratory for Energy Conversion

NGV nozzle guide vane/ stator 1

RS Rotor-stator

SST shear stress transport model

ZnSe Zinc Selenide

\section{Acknowledgements}

This work has been funded by the Federal Ministry for Economic Affairs and Energy via the funding program Luftfahrtforschungsprogramm V, MTU Aero Engines AG and Siemens AG. The authors greatly acknowledge the financial support and the permission to publish. The responsibility for the content of the publication rests with the authors. 


\section{Funding sources}

Siemens AG (Schmid), MTU Aero Engines AG (Lutum, Lecoq), German Federal Ministry of Economic Affairs and Energy.

\section{Competing interests}

Dominic D. Hänni declares that he has no conflict of interest. Rainer Schädler declares that he has no conflict of interest. Reza S. Abhari declares that he has no conflict of interest. Anestis I. Kalfas declares that he has no conflict of interest. Gregor Schmid declares that he has no conflict of interest. Ewald Lutum delcares that he has no conflict of interest. Nicolas Lecoq declares that he has no conflict of interest.

\section{References}

Abhari R. S., Guenette G. R., Epstein A. H., and Giles M. B. (1992). Comparison of time-resolved turbine rotor blade heat transfer measurements and numerical calculations. Journal of Turbomachinery. 114: 818-827. https://doi.org/10.1115/1.2928035

Aulich M. and Siller U. (2011). High-Dimensional Constrained Multiobjective Optimization of a Fan Stage. 1185-1196. https://doi.org/ $10.1115 /$ GT2011-45618

Behr T., Kalfas A. I., and Abhari R. S. (2006). Unsteady flow physics and performance of a one-and-1/2-stage unshrouded high work turbine. Journal of Turbomachinery. 129: 348-359. https://doi.org/10.1115/1.2447707

Blair M. F. (1992). An experimental study of heat transfer in a large-scale turbine rotor passage. Turbo Expo: Power for Land, Sea, and Air, Volume 4: Heat Transfer; Electric Power; Industrial and Cogeneration, V004T09A012.

Dunn M. G., Rae W. J., and Holt J. L. (1984). Measurement and analyses of heat flux data in a turbine stage: Part I-description of experimental apparatus and data analysis. Journal of Engineering for Gas Turbines and Power. 106: 229-233. https://doi.org/10.1115/1.3239539

Hartland J. C., Gregory-Smith D. G., Harvey N. W., and \& Rose M. G. (1999). Non-Axisymmetric Turbine End Wall Design: Part II — Experimental Validation. V001T03A050. https://doi.org/10.1115/99-GT-338

Harvey N. W., Rose M. G., Taylor M. D., Shahpar S., Hartland J., and Gregory-Smith D. G. (1999). Non-Axisymmetric Turbine End Wall Design: Part I — Three-Dimensional Linear Design System. V001T03A049. https://doi.org/10.1115/99-GT-337

Jenny P., Abhari R. S., Rose M. G., Brettschneider M., Engel K., and Gier J. (2013). Unsteady rotor hub passage vortex behavior in the presence of purge flow in an axial low pressure turbine. Journal of Turbomachinery. 135: 051022-051022-9. https://doi.org/10.1115/ 1.4007837

Kupferschmied P., Köppel P., Gizzi W., Roduner C., and Gyarmathy G. (2000). Time-resolved flow measurements with fast-response aerodynamic probes in turbomachines. Measurement Science and Technology. 11: 1036. https://doi.org/10.1088/0957-0233/11/7/318

Kwor E. T. and Matteï S. (2001). Emissivity measurements for Nextel Velvet Coating 811-21 between -36 degrees C and 82 degrees C. 15th European Conference on Thermophysical Properties.

Langston L. S. (2001). Secondary flows in axial turbines-A review. Annals of the New York Academy of Sciences. 934: 11-26. https://doi.org/10.1111/j.1749-6632.2001.tb05839.x

Laveau B., Abhari R. S., Crawford M. E., and Lutum E. (2013). High resolution heat transfer measurement on flat and contoured endwalls in a linear cascade. Journal of Turbomachinery. 135(4):041020-041020-9. https://doi.org/10.1115/1.4007725

Laveau B., Abhari R. S., Crawford M. E., and Lutum E. (2014). High resolution heat transfer measurements on the stator endwall of an axial turbine. Journal of Turbomachinery. 137(4):041005-041005-10. https://doi.org/10.1115/1.4028431

Lazzi Gazzini S., Schädler R., Kalfas A. I., and Abhari R. S. (2017a). Infrared thermography with non-uniform heat flux boundary conditions on the rotor endwall of an axial turbine. Measurement Science and Technology. 28(2):025901-025901-15. https://doi.org/10. 1088/1361-6501/aa5174

Lazzi Gazzini S., Schädler R., Kalfas A. I., Abhari R. S., Hohenstein S., et al. (2017b). Effect of purge air on rotor endwall heat transfer of an axial turbine. Journal of the Global Power and Propulsion Society. 1: 211-223. https://doi.org/10.22261/F29ZWY

Lutum E., Cottier F., Crawford M. E., Laveau B., and Abhari R. S. (2015). A computational investigation of the effect of surface roughness on heat transfer on the stator endwall of an axial turbine. Proceedings of the Institution of Mechanical Engineers, Part A: Journal of Power and Energy. 229: 454-464. https://doi.org/10.1177/0957650915594705

Lynch S. P., Sundaram N., Thole K. A., Kohli A., and Lehane C. (2010). Heat transfer for a turbine blade with nonaxisymmetric endwall contouring. Journal of Turbomachinery. 133: 011019-011019-9. https://doi.org/10.1115/1.4000542

Mansour M., Rebholz P. S., Kalfas A. I., and Abhari R. S. (2015). An on-board wireless multi-sensor measurement system for rotating turbomachinery application. Proceedings of International Gas Turbine Congress, Tokyo, November 15-20, 2015.

Moffat R. J. (1988). Describing the uncertainties in experimental results. Experimental Thermal and Fluid Science. 1: 3-17. https://doi. org/10.1016/0894-1777(88)90043-X

Pfau A., Schlienger J., Kalfas A. I., and Abhari R. S. (2003). Unsteady, 3-Dimensional Flow Measurement Using a Miniature Virtual 4 Sensor Fast Response Aerodynamic Probe (FRAP). 307-315. https://doi.org/10.1115/GT2003-38128

Regina K., Kalfas A. I., and Abhari R. S. (2014). Experimental investigation of purge flow effects on a high pressure turbine stage. Journal of Turbomachinery. 137: 041006-041006-8. https://doi.org/10.1115/1.4028432

Roy A., Jain S., Ekkad S. V., Ng W., Lohaus A. S., et al. (2017). Heat transfer performance of a transonic turbine blade passage in the presence of leakage flow through upstream slot and mateface gap with endwall contouring. Journal of Turbomachinery. 139: 121006-121006-11. https://doi.org/10.1115/1.4037909

Schuepbach P., Abhari R. S., Rose M. G., Germain T., Raab I., and Gier J. (2008). Improving Efficiency of a High Work Turbine Using Non-Axisymmetric Endwalls: Part II-Time-Resolved Flow Physics. 1121-1133. https://doi.org/10.1115/GT2008-50470 
Schuepbach P., Abhari R. S., Rose M. G., and Gier J. (2010). Influence of rim seal purge flow on the performance of an endwall-profiled axial turbine. Journal of Turbomachinery. 133: 021011-021011-10. https://doi.org/10.1115/1.4000578

Sharma O. P. and Butler T. L. (1987). Predictions of endwall losses and secondary flows in axial flow turbine cascades. Journal of Turbomachinery. 109: 229-236. https://doi.org/10.1115/1.3262089

Snedden G., Dunn D., Ingram G., and Gregory-Smith D. (2009). The Application of Non-Axisymmetric Endwall Contouring in a Single Stage, Rotating Turbine. 831-840. https://doi.org/10.1115/GT2009-59169

Thrift A. A., Thole K. A., and Hada S. (2012). Effects of orientation and position of the combustor-turbine interface on the cooling of a vane endwall. Journal of Turbomachinery. 134: 061019-061019-10. https://doi.org/10.1115/1.4004817 